\title{
Correlates of Physical Activity Based on the Social Ecological Model: A Meta-Analysis
}

\author{
Youngho Kim', Jonghwa Lee ${ }^{2}$, Soojin Kang ${ }^{3, *}$ \\ ' Department of Sport Science, Seoul National University of Science and Technology, Seoul, Republic of Korea \\ 2 Department of Physical Education, Seoul National University, Seoul, Republic of Korea \\ ${ }^{3}$ Department of Sport Science, Seoul National University of Science and Technology, Seoul, Republic of Korea
}

Received: August 19, 2021

Accepted: September 29, 2021

Published online: January 31, 2022

Keywords:

Meta-analysis

Physical Activity

Social Ecological Model
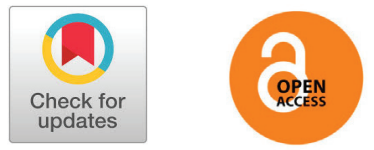

\section{ABSTRACT}

OBJECTIVES The current study aimed to examine the existing literature carried out in Korea regarding physical activity and its related factors based on the social ecological model (SEM).

METHODS A systematic search of relevant literature published from March 2011 to December 20200 was conducted through RISS, KISS, KCI, DBpia, and SEMANTIC SCHOLAR. A total of 9 studies met the inclusion criteria. The selected articles were all of high quality as assessed using the Mixed Methods Appraisal Tool.

RESULTS Results indicated that all SEM constructs were significantly correlated with physical activity. In specific, self-efficacy showed highest correlation effect size with $0.41(95 \% \mathrm{Cl}=0.33 \sim 0.49$ ), followed by pros with $0.26(95 \% \mathrm{Cl}=0.23 \sim 0.30)$, quality of exercise facilities with $0.25(95 \% \mathrm{Cl}=0.19 \sim 0.32)$, accessibility of exercise facilities with $0.23(95 \% \mathrm{Cl}=0.20 \sim 0.26)$, friend support with $0.21(95 \% \mathrm{Cl}=0.15 \sim 0.28)$, and family support with $0.20(95 \% \mathrm{Cl}=0.03 \sim 0.38)$.

CONCLUSIONS The current study was the first attempt applying a meta-analysis to investigate the existing research publications targeted at Korean physical activity based on the SEM. More importantly, the study suggests the direction of evidence-based research by synthesizing studies that applied the SEM to physical activity conducted in Korea and providing practical data to establish strategies to promote physical activity based on the SEM in the future.

( T) The Asian Society of Kinesiology and the Korean Academy of Kinesiology

\section{서론}

2020년 2월부터 시작된 코로나 바이러스 (COVID-19)는 전 세계 모든 사람들을 절망과 공포로 몰아넣고 있으며, 각 국가의 사회, 경제, 문화 모든 영역 의 기능이 제한되고, 경제적 수준이나 성별 및 연령에 관계없이 사망과 질병 발생의 가장 큰 원인으로 작용하 고 있다. 무엇보다도 COVID-19으로 인한 팬데믹은 우 리 생활에서 신체활동 참여를 급격하게 감소시켰으며,

*Correspondence: Soojin Kang, Department of Sport Science, Seoul National University of Science and Technology, Gongneung-ro 232, Nowon-gu, Seoul, Republic of Korea; Tel: +82-02-970-6369; E-mail: eostm2000@naver.com
이로 인해 여러 가지 부정적인 신체적, 정신적 건강문 제가 빠르게 증가하고 있다 $[1,2]$.

행동의학 및 운동심리학의 관점에서 신체활동 참여 를 증진시키기 위해서는 신체활동을 결정하는 다양한 요인들을 분석해 볼 필요가 있다. 즉, 개인의 신체활동 은 그들의 사고, 동기, 자신감, 인지정도, 믿음 등의 심 리행동적 요인, 가족 및 친구의 지지, 사회규범, 사회적 연결망 등의 사회환경적 요인, 그리고 개인이 살고 있 는 지역의 운동시설의 유무 및 접근 용이성, 운동시설 의 질적 수준과 안전성 등과 같은 물리환경적 요인들과 상호 복합적인 인과적 관계를 가지고 있다 [3,4]. 이와 
같은 여러 가지 다양한 요인들이 어떻게 신체활동에 영 향을 미치는지를 보다 더 잘 이해하기 위해서는 과학적 인 근거와 경험적 결과를 기반으로 개발된 이론 및 모 형을 적용하여 신체활동을 연구하는 것이 필수적이라 고 할 수 있다.

Bronfenbrenner의 생태체계이론(ecological system theory)을 기초로 한 사회생태모형 (social ecological model: SEM)은 포괄적인 관점에서 신체활 동에 영향을 주는 변인들을 설명하는 효과적인 개념적 틀로 인식되고 있다 [5]. 이 모형에 따르면, 신체활동에 영향을 주는 요인으로 개인적, 사회문화적, 물리환경적 변인들이 포함된다 [6]. 사회생태모형을 적용한 선행연 구들을 요약해 보면, 높은 자기효능감을 가진 사람들은 효능감이 낮은 사람들에 비해 건강에 대한 신체활동의 효과에 강한 믿음을 가지고 있으며, 따라서 신체활동에 더욱 더 적극적으로 참여하는 것으로 나타났다 [7]. 또 한, 친구와 가족들의 지지는 모든 연령 집단에 걸쳐 폭 넓게 운동참여에 지속적으로 영향을 주는 것으로 보고 되었고, 친구나 가족으로부터의 낮은 사회적 지지는 운 동참여율이 낮아지는 것과 관련이 있는 것으로 나타났 다 [8]. Carron, Hausenblas, Estabrooks는 물리환경 적 요인이 신체활동 참가에 미치는 영향을 연구한 논문 들을 메타 분석한 결과, 날씨, 지역사회 환경, 운동시설 접근용이성에 대한 지각, 운동시설에 대한 실제적 접근 가능성 등이 신체활동의 지속에 유의한 영향을 미치는 것으로 보고하였다 [9].

지금까지 많은 연구들이 사회생태모형을 적용하여 신체활동을 설명하는데 있어 사회생태모형 구성변인의 유의성을 확인하였다. 그러나 이들 연구들은 대부분 서 양에서 수행되었으며, 한국인을 대상으로 수행된 연구 는 매우 부족한 실정이다. 더군다나, 한국에서 발표된 연구들은 연구참여자의 특성, 표본 크기, 연구방법 등에 있어 상이하며, 또한, 신체활동에 대한 사회생태모형 구 성변인의 영향력에 있어 일관된 결과를 보이지 않고 있 다. 따라서, 한국인들의 신체활동에 유의한 영향을 주는 여러 가지 변인들을 확인하고, 이를 활용하여 효과적인 신체활동증진 전략을 개발하기 위하여 본 연구는 사회 생태모형을 적용하여 한국에서 수행된 연구를 대상으 로 메타분석을 실시하여 신체활동에 영향을 미치는 사 회생태모형의 구성변인에 대한 효과크기를 확인하였다.

\section{연구방법}

\section{1. 문헌검색}

문헌검색은 2011년부터 2020년까지 10년간 국내· 외에서 출판된 문헌을 대상으로 하였다. 문헌검색을 위 한 학술 데이터베이스는 학술연구정보서비스 (RISS), 한 국학술정보 (KISS), 한국학술지인용색인 (KC), 국회전 자도서관, 누리미디어 DBpia, SEMANTIC SCHOLAR 를 이용하였다. 분석을 위한 문헌검색은 각 학술 데이 터베이스에서 '사회생태모형'을 검색하고 결과 내 재검 색에서 '신체활동'을 검색하였으며, 국외 학술 데이터 베이스에서는 'social ecological model and physical activity and Korean' 을 검색하여 사회생태모형 연구 에서 신체활동과 관련된 연구를 검색하였다.

\section{2. 선정기준}

신체활동, 사회생태모형 구성변인 간의 상관관계에 대한 메타분석을 위해 다음과 같은 선정기준을 설정하 였다. 첫째, 한국인을 대상으로 연구한 문헌만 선정하였 다. 둘째, 신체활동, 사회생태 변인간의 상관관계를 분 석한 문헌을 선정하였다. 셋째, 메타분석을 위한 신체활 동, 사회생태 변인 간의 상관계수(r)와 참여자 수(n)가 제시된 문헌으로 선정하였다. 넷째, 학위논문을 학술논 문으로 출판한 경우 학술논문을 우선으로 하되 학술논 문으로 출판하는 과정에서 메타분석에 필요한 연구결과 가 누락되었을 경우 학위논문으로 선정하였다. 다섯째, 문헌의 연구설계 유형은 조사연구로 하였다. 여섯째, 학 술 데이터베이스(온라인)에서 열람할 수 없거나 비공개 문헌은 제외하였다.

\section{3. 선정과정}

분석대상 문헌 선정을 위해 각 학술 데이터베이스에서 검색 한 결과, 학술연구정보서비스에서 학술논문 52편, 학위논문 141 편, 보고서 23편, 한국학술정보에서 학술논문 16 편, 보고서 1편, 한국학술지인용색인에서 학술논문 40편, 국회전자도서관에서 학 술논문 7편, 학위논문 3편, 보고서 1편, 누리미디어에서 학술논 문 24편, 보고서 1편, SEMANTIC SCHOLAR에서 학술논문 213 편이 검색되어 총 522 편이 검색되었다. 이 중에서 중복되는 학술 논문 84 편, 학위논문 2 편, 보고서 1편을 제외하였고, 제목과 초록 으로 분류한 결과, 학술논문 258 편, 학위논문 134 편, 보고서 24 편을 제외하였다. 그리고 마지막으로 문헌을 세부적으로 검토한 결과, 중복 게재 2 편, 측정 변인 불일치 3 편, 분석 방법 불일치 5 
편을 제외하여 최종적으로 학술논문 4편과 학위논문 4편(박사학 위논문 1 편, 석사학위논문 3 편), 보고서 1 편이 선정되었다. 분석 대상 문헌 선정과정은 <Figure 1>과 같다.

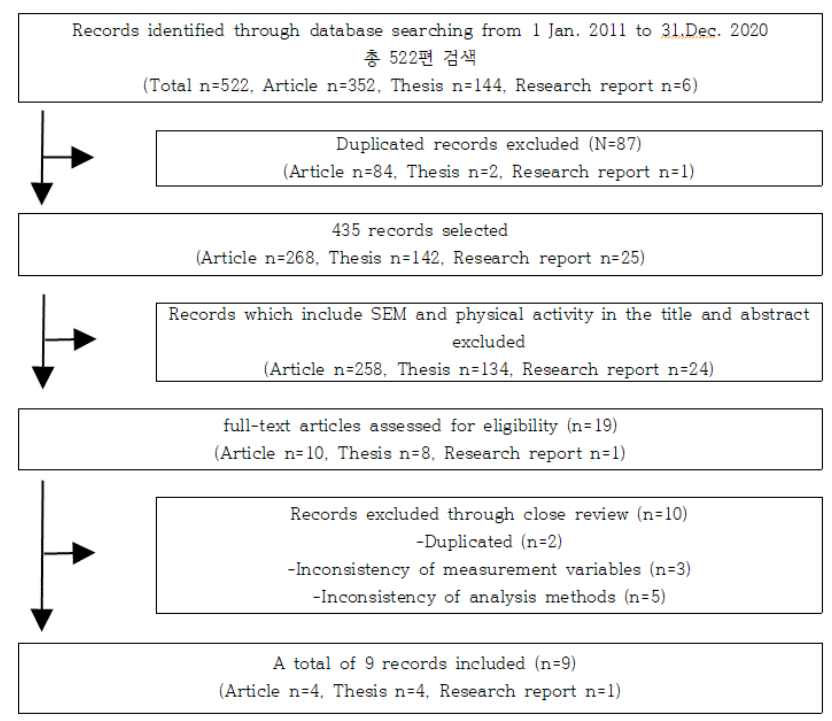

Figure 1. Flowchart outlining the study selection process

\section{4. 자료코딩}

분석대상으로 선정된 9편의 문헌을 코딩하기 위해 문헌의 저자, 출판연도, 출판유형, 연구참여자의 수, 대 상, 그리고 신체활동, 사회생태 변인 간 상관계수의 수 를 코딩하였다. 변인은 신체활동, 자기효능감, 운동이득, 운동장애, 친구지지, 가족지지, 물리적 환경으로 21 개 의 상관관계를 분석하였다. 메타분석을 하기 위해서는 코딩을 위한 명확한 기준이 제시되어야 하고, 코딩하는 연구자들 간의 합의 및 신뢰성이 검증되어야 한다. 이 를 위해 스포츠심리학 박사 2 명이 최종 선정된 문헌의 자료를 코딩하였다. 코딩 과정에서 연구자 간 불일치가 발생했을 경우, 1 인의 스포츠심리학 전공 교수의 자문 을 받아 협의하였다.

\section{5. 자료분석}

메타분석을 위해 R 프로그램(ver. 4.0.5)의 메타분 석 패키지를 활용하였다. 사회생태 변인 간의 상관계수 (r)와 참여자 수 $(n)$ 를 이용하여 효과크기를 추출한 후 Fisher's Z값으로 변환하였다. 정규분포를 더 따르는 Fisher's Z값은 표준화된 값이기 때문에 표본수에 상관 없이 연구 간의 효과 크기 비교가 가능하다 [10]. 다양 한 수준의 개별연구에 대해 좀 더 안정된 통계치를 얻기 위해 역분산 가중치(inverse variance weight)를 사용
하였다 [11]. 메타분석은 개별연구의 효과크기에 가중 치를 부여해서 전체연구의 효과크기, 즉 평균효과크기 를 산출하고 유의성을 검토하여 효과크기의 의미를 설 명하는 것으로, 상관계수 효과크기의 해석은 .10 이하 이면 작은 효과크기, .25 정도면 중간 정도의 효과크기, .40 이상이면 큰 효과크기로 보았다 [12].

분석대상 문헌의 사회생태 변인 간 동질성과 이질성 을 검증하기 위해 Q값과 $\mathrm{I}^{2}$ 값을 이용하였다. Q값이 클 수록 연구결과들 사이에 효과크기 차이가 있는 것을 의 미하며 [13], I2이 25\%이면 낮은 이질성, $50 \%$ 이면 중 간 정도의 이질성, $75 \%$ 이상이면 매우 큰 이질성을 나 타내는 것으로 판단하였다 [14]. 또한 일반적으로 이 질성에 대한 판단은 총분산에 대한 실제 분산의 비율 $\left(\mathrm{I}^{2}\right)$ 이 $50 \%$ 이상이고 동질성 검증의 유의확률이 .01보 다 작은 경우 효과크기의 이질성은 상당하다고 판단하 였다 [14]. 이질성이 높다고 판단되면 랜덤효과 모형 (random-effects model)을 이용하였고, 이질성이 낮은 것으로 판단되면 고정효과 모형(fixed-effects model) 을 이용하였다. 그리고 각 문헌의 변인 간 비뚤림 위험 평가는 risk of bias graph 통해 평가하였고, 출판편향 은 funnel plot을 활용하였다.

\section{연구결과}

\section{1. 문헌의 일반적 특성}

분석대상으로 선정된 문헌은 2011년부터 2020년 사 이에 출판된 학위논문 4 편, 학술논문 4 편(박사학위논문 1 편, 석사학위논문 3편), 보고서 1편으로 모두 9편이었 다. 연구참여자를 살펴보면 노인을 대상으로 한 문헌이 3 편, 성인을 대상으로 한 문헌이 3편, 대학생을 대상으 로 한 문헌이 1 편, 해군을 대상으로 한 문헌이 1 편, 청소 년을 대상으로 한 문헌이 1편이었다 (연구참여자: 5159, 남: 2,061, 여: 3,098) 〈Table 1>. 또한, 〈Table 2〉는 본 연구에 사용된 각 문헌에서 신체활동과 사회생태모 형의 구성변인(자기효능감, 운동이득, 운동장애, 친구 지지, 가족지지, 운동시설의 접근성, 운동시설의 질) 간 의 상관분석 결과를 보여주고 있다. <Table 2>에 따르 면, 사회생태모형의 모든 구성변인들은 신체활동과 유 의한 상관성이 있는 것으로 나타났으며, 특히, 사회생태 모형의 구성변인들 중, 운동장애 변인은 3 편의 논문에 서 신체활동과 음의 상관성이 있는 것으로 확인되었다. 
Table 1. Studies included in the meta-analysis

\begin{tabular}{|c|c|c|c|c|c|}
\hline Author(year) & Participants(age) & Gender & $\begin{array}{l}\text { Number of } \\
\text { participants }\end{array}$ & Study location & $\begin{array}{l}\text { Number of assessed correlation } \\
\text { coefficient }\end{array}$ \\
\hline $\mathrm{Gu}(2020)$ & Adolescents(-19) & $\begin{array}{l}\text { Male }=752 \\
\text { Female }=842\end{array}$ & 1604 & Seoul, KyoungGi-Do & 15 \\
\hline $\mathrm{Heo}(2014)$ & College students(20-26) & $\begin{array}{l}\text { Male }=153 \\
\text { Female }=203\end{array}$ & 356 & Seoul & 10 \\
\hline Hwang \& Kim(2017) & Older adults(+65) & $\begin{array}{l}\text { Male }=125 \\
\text { Female }=276\end{array}$ & 401 & Seoul & 21 \\
\hline Kang(2011) & Older adults(+65) & $\begin{array}{l}\text { Male }=87 \\
\text { Female }=196\end{array}$ & 283 & Seoul & 21 \\
\hline Kang et al.(2016) & Adults(+30) & $\begin{array}{l}\text { Male }=332 \\
\text { Female }=480\end{array}$ & 812 & Seoul & 21 \\
\hline Kim \& Kosma,(2012) & Older adults(+65) & $\begin{array}{l}\text { Male }=87 \\
\text { Female }=203\end{array}$ & 290 & Seoul & 21 \\
\hline Kim et al.(2013) & Adults $(+20)$ & $\begin{array}{l}\text { Male }=182 \\
\text { Female }=169\end{array}$ & 352 & Seoul & 21 \\
\hline Kim et al.(2014) & Adults $(+20)$ & $\begin{array}{l}\text { Male }=330 \\
\text { Female }=549\end{array}$ & 879 & Seoul & 21 \\
\hline $\operatorname{Roh}(2011)$ & $\operatorname{Navy}(+20)$ & $\begin{array}{l}\text { Male }=179 \\
\text { Female }=5\end{array}$ & 184 & Jinhae & 21 \\
\hline
\end{tabular}

Table 2. Relationships between the SEM constructs and physical activity

\begin{tabular}{|c|c|c|c|c|c|c|c|c|c|}
\hline & $\begin{array}{l}\text { Kang } \\
\text { (2011) }\end{array}$ & $\begin{array}{l}\text { Roh } \\
\text { (2011) }\end{array}$ & $\begin{array}{l}\text { Kim \& Kosma } \\
\text { (2012) }\end{array}$ & $\begin{array}{l}\text { Kim et al. } \\
(2013)\end{array}$ & $\begin{array}{l}\text { Heo } \\
(2014)\end{array}$ & $\begin{array}{l}\text { Kim et al. } \\
(2014)\end{array}$ & $\begin{array}{l}\text { Kang et al. } \\
\text { (2016) }\end{array}$ & $\begin{array}{l}\text { Hwang \& } \\
\text { Kim (2017) }\end{array}$ & $\begin{array}{l}\mathrm{Gu} \\
(2020)\end{array}$ \\
\hline \multicolumn{10}{|c|}{ Psychological variables } \\
\hline Self-efficacy & Cor(+) & NC & Cor(+) & Cor $(+)$ & Cor(+) & Cor(+) & Cor $(+)$ & Cor $(+)$ & Cor(+) \\
\hline Perceived benefits & Cor $(+)$ & Cor $(+)$ & Cor(+) & NC & Cor(+) & Cor(+) & Cor $(+)$ & Cor $(+)$ & $\operatorname{Cor}(+)$ \\
\hline Perceived barriers & Cor(-) & Cor(-) & Cor(-) & Cor(-) & NC & Cor(+) & Cor $(+)$ & Cor $(+)$ & $\operatorname{Cor}(+)$ \\
\hline \multicolumn{10}{|l|}{ Social variables } \\
\hline Friends support & Cor(+) & NC & Cor(+) & Cor(+) & Cor(+) & Cor(+) & Cor(-) & $\operatorname{Cor}(+)$ & Cor(+) \\
\hline Family support & Cor $(+)$ & NC & Cor $(+)$ & Cor(+) & Cor(+) & Cor(+) & Cor $(+)$ & Cor $(+)$ & Cor(+) \\
\hline \multicolumn{10}{|c|}{ Physical environmental variables } \\
\hline Physical environment & $\operatorname{Cor}(+)$ & $\operatorname{Cor}(+)$ & $\operatorname{Cor}(+)$ & $\operatorname{Cor}(+)$ & Cor(+) & $\operatorname{Cor}(+)$ & $\operatorname{Cor}(+)$ & Cor(+) & $\operatorname{Cor}(+)$ \\
\hline
\end{tabular}

\section{2. 신체활동과 사회생태모형 구성변인간의 상관관계 효과크기}

<Table 3>은 본 연구에 선정된 문헌에서 신체활동 과 사회생태모형의 구성변인의 상관관계 효과크기를 대 상으로 메타분석한 결과를 보여주고 있다. 분석의 초기 단계에서 각 문헌들의 동질성과 이질성을 평가하였다. 이를 통해 사회생태모형의 구성변인 중, 운동이득 변인 과 운동시설의 접근성 변인은 이질성이 낮게 나타나 고 정효과 모형으로 분석을 하였으며, 다른 변인들은 이질
성이 높게 나타나 랜덤효과 모형으로 분석하였다.

<Table 3>에 따르면, 사회생태모형의 모든 구성변인 들과 신체활동 변인의 상관관계 효과크기는 유의한 것 으로 나타났다. 구체적으로, 신체활동과 자기효능감 변 인의 상관관계 효과크기는 $0.41(95 \% \mathrm{CI}=0.33 \sim 0.49)$ 로 가장 큰 효과크기를 보였으며, 그 다음으로 운동이 득 0.26(95\% CI=0.23 0.30), 운동시설의 질 0.25(95\% $\mathrm{CI}=0.19 \sim 0.32)$, 운동시설의 접근성 $0.23(95 \%$ $\mathrm{CI}=0.20 \sim 0.26)$, 친구지지 $0.21(95 \% \mathrm{CI}=0.15 \sim 0.28)$, 
Table 3. Effect sizes of the social ecological model constructs on physical activity

\begin{tabular}{lllllll}
\hline Social ecological constructs & $\mathbf{K}$ & $\mathbf{I}^{\mathbf{2}}$ & $\mathbf{Q}(\mathbf{d f})$ & $\boldsymbol{p}$ & Model & $\boldsymbol{Z}_{\boldsymbol{r}}[\mathbf{9 5} \% \mathbf{C l}]$ \\
\hline Self-efficacy & 8 & 81.6 & $38.12(7)$ & $<0.0001$ & random & $0.41[0.33-0.49]$ \\
Perceived benefits & 7 & 14.8 & $7.04(6)$ & $=0.3168$ & fixed & $0.26[0.23-0.30]$ \\
Perceived barriers & 7 & 93.1 & $87.08(6)$ & $<0.0001$ & random & $-0.03[-0.17-0.10]$ \\
Friend support & 8 & 70.5 & $23.73(7)$ & $=0.0013$ & random & $0.21[0.15-0.28]$ \\
Family support & 8 & 96.3 & $190.53(7)$ & $<0.0001$ & random & $0.20[0.03-0.38]$ \\
Physical environment & 8 & 3.3 & $7.24(7)$ & $=0.4044$ & fixed & $0.23[0.20-0.26]$ \\
\hline
\end{tabular}

$K=$ the number of effect sizes; $\mathrm{Cl}=$ Confidence interval; $d f=$ degree of freedom; $Q=C o c h r a n ' s, I^{2}=$ Inconsistency index

가족지지 0.20(95\% CI=0.03 0.38)의 순으로 효과크 기가 유의한 것으로 보고되었다.

\section{논의}

본 연구는 사회생태모형을 적용하여 한국에서 수행 된 연구를 대상으로 메타분석을 실시하여 신체활동에 영 향을 미치는 사회생태모형의 구성변인에 대한 효과크기 를 확인하였다. 이를 위해 2011년부터 2020년까지 출 판된 연구들 중, 메타분석의 실시 기준을 충족하는 9편 의 문헌이 최종적으로 본 연구에 선정하였다.

본 연구의 가장 핵심적인 연구문제는 한국인들이 신 체활동을 지속하는데 영향을 주는 유의한 요인들을 확 인하는 것이다, 이를 위해 포괄적인 관점에서 신체활동 을 설명하고 있는 사회생태모형을 적용한 선행연구들을 분석하였으며, 그 결과 심리적, 사회적, 물리환경적 변 인들은 모두 신체활동과의 상관관계 효과크기가 중간수 준인 것으로 나타났다. 구체적으로 모든 심리적 변인 ( 자기효능감, 운동이득 및 운동장애)들이 신체활동과 유 의한 상관성이 있는 것으로 나타났다. 특히, 자기효능감 은 청소년, 성인, 노인 등 모든 연령층에서 그들의 신체 활동과 가장 상관성이 높은 변인으로 보고되었으며, 국 외에서 수행된 메타분석 연구들에 의해 지지되고 있다 $[15,16]$. 또한, 본 연구에서 운동이득 및 운동장애 변 인은 신체활동과 관련이 있는 것으로 나타났으며, 여러 국외에서 수행된 연구들에 의해 지지되었다 $[17,18]$. 이러한 결과는 지역 주민들의 신체활동 참여 증진을 통 해 운동이득에 대한 이해와 운동장애에 대한 경각심을 고취시켜 궁극적으로 지역 주민들의 건강상태를 개선하 고 질병을 예방할 수 있는 지역사회 건강증진전략을 개
발하는데 유용하게 적용될 수 있다.

본 연구에서 사회적 변인으로 사회적 지지는 신체활 동과 유의한 상관성이 있는 것으로 나타났다. Smith와 그의 동료들에 따르면, 사회적 지지는 개인을 둘러싼 중요한 사회적 환경 요인 중의 하나로 신체활동을 포함 한 건강행동 참여에 동기를 부여하는데 중요한 변인으 로 인식되고 있다. 구체적으로 본 연구에서 청소년들은 친구의 지지가 그들의 신체활동 참여에 보다 중요한 사 회적 요인이라고 인식하고 있는 반면, 노인들은 가족의 지지가 신체활동과 보다 더 유의한 상관이 있는 것으로 보고하였다 [19]. 이러한 연구결과는 선행연구들에 의 해 폭 넓게 지지되고 있다 [20,21]. 따라서 신체활동을 생활화하고 이를 통해 건강한 삶을 보내기 위해서는 개 인의 주변에 이를 지지해 줄 수 있는 사회적 지지체계 를 형성하는 것이 필요할 것으로 사료된다.

운동심리학 영역에서 사회생태모형이 가지는 유의성 은 신체활동을 설명하는데 있어 사회심리적 변인에 초 점을 두는 전통적인 접근방식을 넘어 물리적 환경변인 을 포함하는 포괄적인 접근방식을 취한다는 것이다. 본 연구의 결과에 따르면, 신체활동 장소 및 시설 등의 물 리적 환경은 신체활동과 통계적으로 유의한 상관이 있 는 변인으로 나타났다. 특히, 운동시설 및 장소의 근접 성 및 질적 상태는 본 연구에 사용된 모든 문헌에서 유 의한 관련성을 보였으며, 이러한 결과는 많은 선행연구 들에 의해 지지되고 있다 [22,23]. 따라서 선행 연구의 메타분석 결과와 본 연구에서 얻어진 결과들을 종합하 면, 규칙적으로 신체활동에 참여하는 사람들일수록 자 신의 신체활동과 관련된 운동시설의 접근성 및 시설의 질적 상태 등의 변인들을 긍정적으로 인식하는 경향을 보이는 것으로 해석될 수 있을 것이다. 특히, 본 연구에 
사용된 문헌들 대부분이 성인들을 대상(노인대상 3편) 으로 수행되었으며, 각 문헌에서 연구참여자들의 인구 통계학적 특성과 무관하게 규칙적으로 신체활동을 하는 비율이 $70 \%$ 이상으로 나타났다. 이와 같은 결과는 규칙 적으로 신체활동에 참여하는 사람들의 경우 신체활동 의 건강상의 이득을 충분히 인지하고 있을 가능성이 높 아 주변 운동시설이나 운동정보에 대한 인식이 매우 높 을 수 있다. 또한 사이클링, 걷기 및 조깅, 볼링, 요가 등 의 여가생활 성격이 강한 신체활동 참여자의 경우 신체 활동의 특성상 물리적 환경의 중요성이 무엇보다도 크 게 영향을 미치는 것으로 판단된다 [24]. 이러한 해석 은 신체활동 증진을 위해서는 개인이 선호하는 신체활 동을 고려한 환경지지적 맞춤식 신체활동 처치전략의 필요성을 강조하는데 중요한 자료가 될 수 있을 것이다.

상기 기술되어진 유의한 연구결과에도 불구하고, 본 연구는 다음과 같은 제한점을 가지고 있다. 또한, 본 연 구는 메타분석을 수행하기 위해 엄격하고 객관적인 연 구방법 및 절차를 준용하여 연구에 사용된 문헌들을 선 정하였다. 이를 통해 총 9편의 연구문헌이 문헌선정 기 준인 연구설계, 측정변인, 분석방법 등의 조건에 부합하 여 본 연구에서 적용되었으나, 본 연구에서 도출된 결과 의 유의성을 객관적으로 지지하는 데에는 충분하지 않 을 수도 있다. 하지만 선행연구에 따르면, 메타분석에 서 연구에 사용되는 연구문헌의 수는 연구자가 통제할 수 있는 것이 아니며, 기술적으로는 2편 이상만 되어도 분석이 가능하다고 보고 있다 [25].

본 연구는 한국인들의 신체활동과 관련된 다양한 요 인들을 확인하기 위하여 포괄적인 관점에서 신체활동 에 초점을 두고 있는 사회생태모형을 적용한 연구문헌 들을 메타분석한 첫 번째 시도이다. 본 연구에서 사회생 태모형은 신체활동을 설명하는데 있어 유의하다는 것이 확인되었다. 하지만, 본 연구에 사용된 연구들 모두 도 시에 거주하는 성인과 노인들로 국한되어 있으므로 앞 으로의 후속연구들은 다른 지역 및 다른 연령층을 대상 으로 사회생태모형을 적용한 신체활동 연구를 확대해 야 할 필요가 있다. 본 연구는 한국에서 수행된 신체활 동에 대해 사회생태모형을 적용한 연구들을 종합하고, 향후 사회생태모형을 기반으로 신체활동을 증진시키는 전략을 수립하기 위한 실질적 자료를 제공함으로써 근 거기반 연구의 방향성을 제시하였다는데 의의가 있다.

\section{Conflict of Interest}

The authors declare no conflict of interests.

\section{Funding}

This work was supported by the Ministry of Education of the Republic of Korea and the National Research Foundation of Korea (NRF-2020S1A5A2A03041894).

\section{References}

1. Goessler KF, Roschel H, Gualano B. Social isolation during the COVID-19 pandemic can increase physical inactivity and the global burden of cardiovascular disease, American Journal of Physiology: Heart and Circulatory Physiology. 2020; 318 (6):441-446.

2. Guo YF, Liao MQ, Cai WL, et al. Physical activity, screen exposure and sleep among students during the pandemic of COVID-19. Science Reports. 2021; 11:111. 8529.

3. Kim YH, Kosma M. Psychosocial and Environmental Correlates of Physical Activity Among Korean Older Adults. Research on Aging. 2012; 35 (6):750-767.

4. Kang SJ, Kim YH. The effect of individual, social, and physical environment variables on older adults' physical activity. Korean Journal of Sport Psychology. 2011; 22 (3):113-124.

5. Kang SJ, Kim YH, Park IK. Causal Relationship among the Social Ecological Variables to Explain Physical Activity. Korean Journal of Sport Psychology. 2016; 27 (2): 53-64.

6. Bronfenbrenner U. Ecological systems theory. Annual of Child Development. 1989; 22: 723-742.

7. Hwang J, Kim YH. Psychological, Social Environmental, and Physical Environmental Variables in Explaining Physical Activity in Korean Older Adults. Journal of Sport Psychology. 2017; 26 (1):83-92.

8. Pis MB. Physical activity social support, self-efficacy, and self-definition in adolescents: A correlational crosssectional comparative study. (Doctoral Dissertation). Retrieved from ProQuest Dissertations and Theses (Accession Order No. AAT 3224723). 2006. 
9. Carron AV, Hausenblas HA, Estabrooks PA. The psychology of physical activity. New York: McGraw-Hill. 2003.

10. Borenstein M, Hedges LV, Higgins JPT, Rothstein HR. Introduction to meta-analysis. West Sussex, UK: Wiley. 2009.

11. Oh SS. Meta-analysis: theory and practice. Seoul: Konkuk University Press. 2002.

12. Cohen J. Statistical power analysis for the behavioral sciences (2nd ed.). Hillsdale, NJ: Lawrence Earlbaum Associates. 1988.

13. Corcoran K, Crusius J, Mussweiler T. Social comparison: Motives, standards, and mechanisms. In D. Chadee (Ed.), Theories in social psychology. Wiley Blackwell, 2011. p119-139.

14. Higgins J, Green S. Cochrane handbook for systematic reviews of interventions version 5.1.0. The codhrane collaboration retrieved from www.cochrane-handbook. org. 2011.

15. Bauman A, Reis R, Sallis J, Wells J, Loos R, Martin B. Correlates of physical activity: Why are some people physically active and others not? The Lancet. 2012; 380:258-271.

16. Sterdt E, Liersch S, Walter U. Correlates of physical activity of children and adolescents: A systematic review of reviews. Health Education Journal. 2014; 73(1):72-89.

17. Biddle S, Aykin A, Cavill N, Foster C. Correlates of physical activity in Youth: A review of quantitative systematic reviews. International Review of Sport and Exercise Psychology. 2011; 4(1):423-434.

18. Van der Horst K, Chin M, Twisk J, Van Mechelen W. A brief review on correlates of physical activity and sedentariness in youth. Medicine \& Science in Sports and Exercise. 2007; 39(8):1241-1250.

19. Smith GL, Banting L, Eime R, O’Sullivan G, van Uffelen JGZ. The association between social support and physical activity in older adults: A systematic review. International Journal of Behavioral Nutrition and Physical Activity. 2017; 14(56):1-21.

20. Laird Y, Fawkner S, Kelly P, McNamee L, Niven A. The role of social support on physical activity behavior in adolescent girls: A systematic review and meta-analysis. International Journal of Behavioral Nutrition and Physical Activity. 2016; 13(79):1-14.

21. Yao C, Rhodes R. Parental correlates in child and adolescent physical activity: A meta-analysis. International Journal of Behavioral Nutrition and Physical Activity. 2015; 12(10):1-38.

22. Ding D, Sallis J, Kerr J, Lee S, Rosenberg D. Neighborhood environment and physical activity among youth: A review. American Journal of Preventive Medicine. 2011; 41(4):442-455.

23. Oliveira A, Moreira C, Abreu S, Mota J, Santos R. Environmental determinants pf physical activity in children: A systematic review. Archives of Exercise in Health and Disease. 2014; 4(2):254-261.

24. Hu DL, Zhou S, Crowley-McHattan Z, Liu ZY. Factor that influence participation in physical activity in school-aged children and adolescents: A systematic review from the social ecological model perspective. International Journal of Environmental research and Public Health. 2021 18(6):3147.1-22.

25. Valentine JC, Pigott TD, Rothstein HR. How many studies do you need? A primer on statistical power for metaanalysis. 2010 35(2):215-247. 